\title{
The colonization of pyrethroid resistant strain from wild Anopheles sinensis, the major Asian malaria vector
}

Guoding Zhu ${ }^{1,2}$, Huayun Zhou', Julin Li ${ }^{1}$, Jianxia Tang ${ }^{1}$, Liang Bai', Weiming Wang ${ }^{1}$, Yaping Gu', Yaobao Liu', Feng Lu', Yuanyuan Cao ${ }^{1}$, Chao Zhang ${ }^{1}$, Sui Xu' ${ }^{1}$, Jun Cao ${ }^{1,3^{*}}$ and Qi Gao ${ }^{1,2^{*}}$

\begin{abstract}
Background: Anopheles sinensis is one of the most important malaria vectors in Asian countries. The rapid spread of insecticide resistance has become a major obstacle for insecticide-based strategies for vector control. Therefore, it is necessary to prepare an insecticide-resistant strain of An. sinensis to further understand the insecticide resistance mechanisms in this species to facilitate genetic approaches to targeting the insecticide-resistant population of this important malaria vector.
\end{abstract}

Methods: An. sinensis mosquitoes were collected from regions where pyrethroid resistance had been reported. The mosquitoes were subjected to continuous pyrethroid selection after species confirmation, and the forced copulation method was used to increase the mating rate. In addition, the knockdown-resistance ( $k d r)$ mutation frequencies of each generation of An. sinensis were measured; and the metabolic enzyme activities of cytochrome P450 monoxygenases (P450s) and glutathione S-transferases (GSTs) were detected.

Results: The identification of field-captured An. sinensis was confirmed by both morphological and molecular methods. The population of An. sinensis exhibited stable resistance to pyrethroid after continuous generations of pyrethroid selection in the laboratory with high kdr mutation frequencies; and elevated levels of both P450s and GSTs were significantly found in field selected populations comparing with the laboratory susceptible strain. So far, the colonised strain has reached its eleventh generation and culturing well in the laboratory.

Conclusions: We colonised a pyrethroid-resistant population of An. sinensis in the laboratory, which provides a fundamental model for genetic studies of this important malaria vector.

Keywords: Anopheles sinensis, Pyrethroid, Insecticide resistance

\section{Background}

Anopheles sinensis (Diptera, Culicidae) is one of the major malaria vector mosquitoes in China and other Asian countries [1-4]. This species is an important member of the An. hyrcanus group (Diptera, Culicidae) with similar morphology [5]. Increased attention has been paid to this species due to its wide distribution, high abundance and modest susceptibility to malaria parasites reported in previous studies $[6,7]$.

\footnotetext{
* Correspondence: caojuncn@hotmail.com; gaoqi54@hotmail.com 'Key Laboratory of Parasitic Disease Control and Prevention (Ministry of Health), and Jiangsu Provincial Key Laboratory of Parasite Molecular Biology, Jiangsu Institute of Parasitic Diseases, Wuxi, Jiangsu Province, People's Republic of China

Full list of author information is available at the end of the article
}

Vector control is defined by the World Health Organization (WHO) as one of the four basic and most effective measures to prevent malaria transmission and remains a component of malaria control strategies, including in the elimination stage [8]. The intervention based on indoor residual spraying (IRS) is the most widely adopted method in almost all regions in China at risk of malaria transmission. Pyrethroids are often used for IRS due to their relative long persistence and low toxicity in comparison with the other three major classes of available insecticides [9]. However, the substantial increase in pyrethroid-based malaria vector control in the past decade has resulted in the rapid spread of resistance among malaria vectors. Furthermore, the widespread use of these compounds for 
agricultural purposes has further accelerated the development of insecticide resistance [10]. High-level resistance to pyrethroids has been reported in An. sinensis, especially in central China, in recent years, which has placed current national efforts of malaria elimination at risk [11]. As a result, improvements to the surveillance of and response to insecticide resistance in An. sinensis in China are urgently needed and must be based on a greater understanding of the molecular mechanisms underlying this resistance.

Genetic approaches, such as genetic linkage studies and QTL mapping [12-14], are useful tools for understanding insecticide resistance mechanisms and developing a method for monitoring insecticide resistance in both the susceptible and resistant strains. Our laboratory has successfully colonised the susceptible $A n$. sinensis strain; more than five hundred generations of this strain have never been exposed to any insecticide, dating back to the 1980s. To prepare an insecticide-resistant strain in the laboratory, in 2013, we collected wild $A n$. sinensis from areas with reports of high resistance levels in recent years [15] for use in breeding field-derived, insecticide-resistant $A n$. sinensis in the laboratory. The knockdown-resistant $(k d r)$ mutation frequencies among continuous generations, and the metabolic enzyme activities were also investigated.

\section{Methods}

\section{Mosquito colonisation}

From June to July in 2013, living engorged female anopheline mosquitoes (F0) were captured gently with a mouth aspirator in pigsties near a rice field in Yixing County $\left(119^{\circ} 38^{\prime} \mathrm{E}, 31^{\circ} 16^{\prime} \mathrm{N}\right)$, Jiangsu Province, China, where Plasmodium vivax has been the only prevalent species of malaria in recent years (Figure 1). Subsequently, all of the mosquitoes were transported to the insectary of the Key Laboratory on Technology for Parasitic Disease Control and Prevention, Ministry of Health, Jiangsu Institute of Parasitic Diseases (JIPD) in Wuxi, Jiangsu Province, China. The insectary is maintained at $26 \pm 1^{\circ} \mathrm{C}, 70-80 \%$ relative humidity with a $12 \mathrm{~h}$ day/night lighting regime, and the mosquitoes were provided with $10 \%(\mathrm{w} / \mathrm{v})$ glucose in water. Distilled water-saturated filter paper was placed in the mosquitoes' cage, and the females were allowed to oviposit. Subsequently, the eggs were morphologically identified to avoid potential contamination with the other similar anopheline mosquitoes of the An. hyrcanus group, such as An. anthropophagus (Diptera, Culicidae), which closely resembles An. sinensis in the adult stage but differs morphologically in the egg stage. All families identified as An. sinensis were pooled, and larvae were reared to adults (first generation, F1). The larvae were fed finely ground tropical fish food (TetraMin, Germany). Newly emerged female and male adults were placed in separate containers to ensure that mating did not take place prior to exposure to insecticides. Randomly selected F1 adults were identified using the $r D N A I_{T S S_{2}}$ based method to further confirm the species' identity [16].

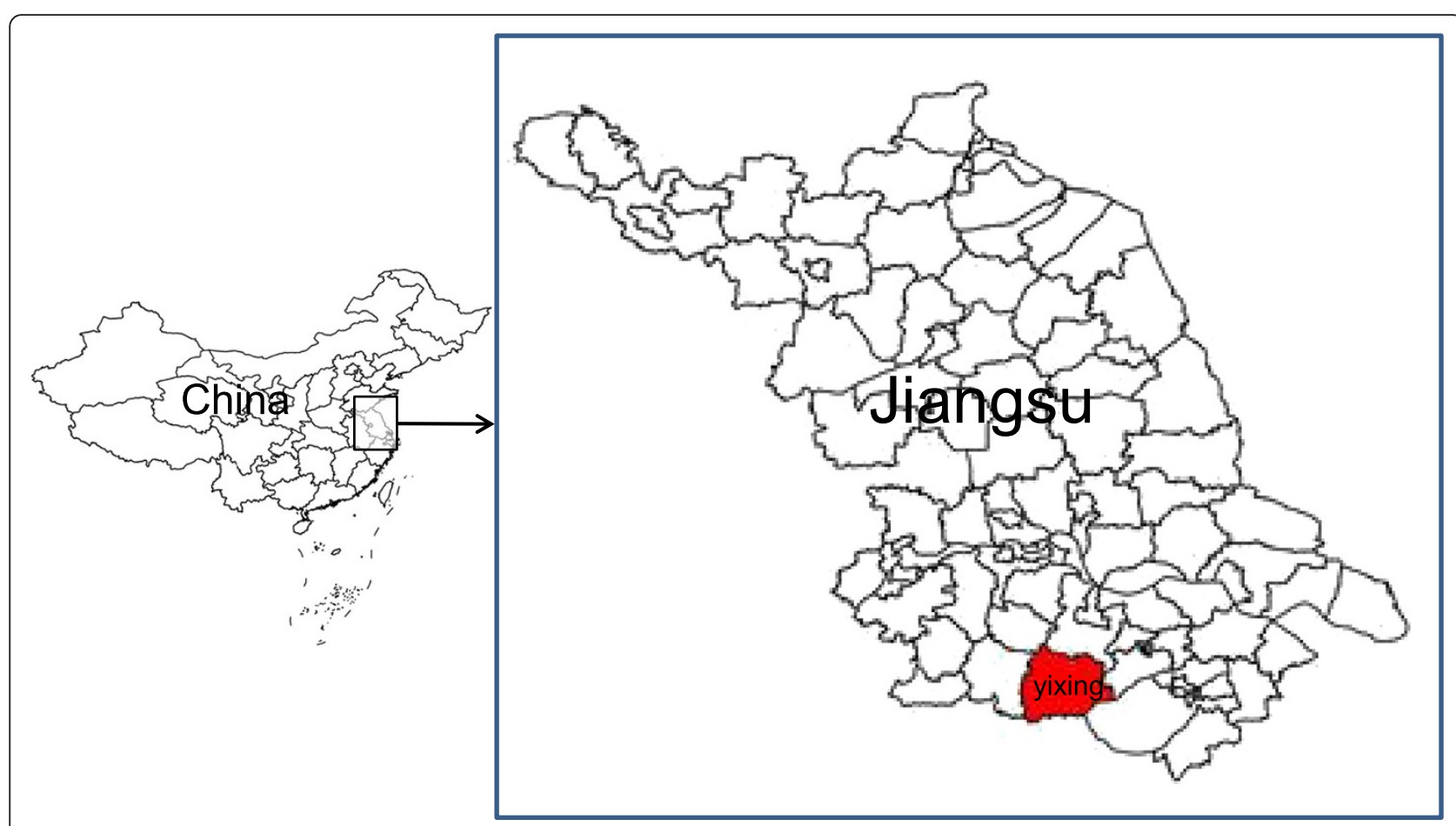

Figure 1 Study site for field mosquito collection in 2013. 


\section{Pyrethroid-resistance selection}

All the 3- to 5-day-old newly emerged female and male adults, approximately twenty-five mosquitoes per exposure tube, from each generation were exposed to $0.05 \%$ deltamethrin-treated papers for $1 \mathrm{~h}$ according to the standard WHO bioassay procedure (WHO, 1998). WHO (2013) criteria were used to classify the resistance status of the tested mosquito population after $24 \mathrm{~h}$ recovery from the insecticide [17]. All of the male and female survivors $24 \mathrm{~h}$ post-exposure were placed in separate cages, and the females were left for blood-feeding followed by forced mating with the male survivors of similar age. After two days, water was added to the filter paper, and female mosquitoes were allowed to oviposit; second bloodmeals were offered to allow the females to oviposit again [18]. Eggs from each generation were reared to adults and selected for resistance to deltamethrin in the same manner until a stable resistance level was reached, after which insecticide selection with a generation interval was used.

\section{Forced mating}

To overcome the obstacle of the low rate of mating resulting from the shift to the new environment among the field-collected mosquitoes in the laboratory, the forced mating method based on MR4 was used to increase the mating rate [19]. Briefly, the female mosquitoes living after $24 \mathrm{~h}$ recovery from the exposure to deltamethrin were blood-fed with an anaesthetised mouse. The engorged female mosquitoes were anaesthetised by ethyl ether $4-5 \mathrm{~h}$ post-blood-feed; the well-fixed males were decapitated to overcome the potential innate inhibition of their copulatory muscles. The males were placed at an angle venter-to-venter with the female until the males clasped the females and mated successfully.

\section{gDNA extraction and $k d r$ genotype identification}

The total genomic DNA of individual mosquitoes was extracted using QIAGEN DNeasy 96 Kits according to the manufacturer's instructions. Briefly, one or two mosquito legs were added to a mixture of $100 \mu \mathrm{L}$ of Tissue Lysis Solution and $10 \mu \mathrm{L}$ of Proteinase $\mathrm{K}$ and were incubated at $55^{\circ} \mathrm{C}$ for $10 \mathrm{~min}$ and $95^{\circ} \mathrm{C}$ for $10 \mathrm{~min}$. Next, $100 \mu \mathrm{L}$ of Neutralization Solution $\mathrm{T}$ was added to the mixture, which was then centrifuged at $17,900 \times \mathrm{g}$ for $3 \mathrm{~min}$. The supernatant was transferred to a new tube and stored at $-30^{\circ} \mathrm{C}$ for species identification and kdr detection. The DNA extracts of randomly selected F1 mosquitoes were amplified for species determination using an $r D N A-I T S_{2}$ based method. PCR products were visualised under ultraviolet light after electrophoresis using $2 \%$ agarose gel stained with ethidium bromide. DNA extracts of female mosquitoes from F0 to F11 were genotyped individually using a recently developed TaqMan-MGB probe assay for $k d r$ mutation detection at codon position 1014 of the para-type sodium channel $[20,21]$ and run on a LightCycler 480 qPCR thermal cycler (Roche). The PCR conditions were an initial denaturation step of $10 \mathrm{~min}$ at $95^{\circ} \mathrm{C}$ followed by 40 cycles of $95^{\circ} \mathrm{C}$ for $10 \mathrm{~s}$ and $65^{\circ} \mathrm{C}$ for $15 \mathrm{~s}$.

\section{Metabolic enzyme activity detection}

The 3-4 days post emergence female mosquitoes alive $24 \mathrm{~h}$ after $60 \mathrm{~min}$ exposure to insecticide from F11, and the mosquitoes knocked down from colonized laboratory susceptible populations during the 60 min exposure were selected, to ensure only the fresh mosquitoes were immediately tested for metabolic enzyme activities. The selected individual mosquitoes were homogenized with phosphate $\mathrm{KPO}_{4}$ buffer $(0.25 \mathrm{M}, \mathrm{pH} 7.2)$, and diluted by adding phosphate buffer, and then the supernatant was used to test the enzyme activities of cytochrome P450 monoxygenases (P450s) and glutathione S-transferases (GSTs) based on our previously published protocol [22]. Mean absorbance values for each tested mosquito and enzyme were converted into enzyme activity and standardized based on the total protein amount. All measurements were performed in duplicate.

\section{Statistical analysis}

The Chi-square test was used to compare the mortalities between the sexes and the generations. The $t$-test was used to determine whether monooxygenases and GST activity varied between the laboratory strain and the field An.sinensis mosquitoes.

\section{Ethical approval of animal use}

The animal experiments were approved by the Jiangsu Institutional Animal Care and Use Committee (IACUC), according to the guideline of administration of lab animals issued by the Ministry of Science and Technology (Beijing, China). All animal procedures were approved by the Institutional Review Board (IRB00004221) of Jiangsu Institute of Parasitic Diseases (Wuxi, China).

\section{Results}

\section{Mosquito species confirmation and colonisation}

From June to July, we collected more than 2000 engorged anopheline mosquitoes. All of the batches of eggs laid by the parents (F0) were morphologically confirmed as An. sinensis, which exhibit a relatively wider float than the considerably narrower one in An. anthropophagus (Figure 2). Anopheles. anthropophagus has historically been distributed from limited areas in Jiangsu Province. The randomly selected F1 adults were further confirmed as An. sinensis using the $r D N A-I T S_{2}$ method [16].

\section{Pyrethroid resistance selection}

Male and female F1 An. sinensis mosquitoes were separately tested with a standard WHO bioassay, and the resistance status of both sexes was classified as "Resistant". 


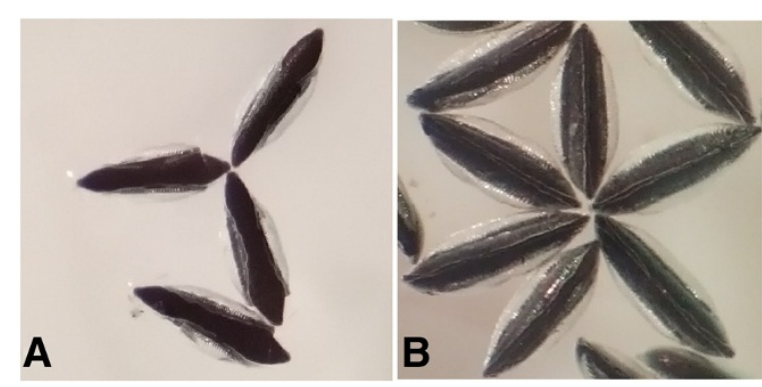

Figure 2 Morphological characteristics of the eggs of An. sinensis and An. anthropophagus, observed under a normal dissection microscope. (A). The wide float in An. sinensis. (B). The narrow float in An. anthropophagus.

As shown in Table 1 , the mortality decreased significantly in males $\left(\chi^{2}=51.79, v=2, p<0.01\right)$ and females $\left(\chi^{2}=28.07\right.$, $v=2, p<0.01)$ after the deltamethrin selection process from F1 to F3. After F4, both the male and female populations exhibited zero mortality. The females were more resistant to the deltamethrin treatment than the males in the F1 $\left(\chi^{2}=\right.$ $16.98, v=1, p<0.01)$ and F2 $\left(\chi^{2}=22.52, v=1, p<0.01\right)$ generations; however, no significant difference was observed in

Table 1 Mortality upon exposure to deltamethrin in An. sinensis populations

\begin{tabular}{llll}
\hline Population & & Sample size $(\mathbf{n})$ & Mortality \pm SE (\%) \\
\hline Field-F1 & Male & 570 & $14.08 \pm 0.48$ \\
& Female & 470 & $6.21 \pm 0.21$ \\
Field-F2 & Male & 469 & $11.86 \pm 2.21$ \\
& Female & 495 & $3.66 \pm 0.98$ \\
Field-F3 & Male & 402 & $0.84 \pm 0.27$ \\
& Female & 510 & $0.27 \pm 0.12$ \\
Field-F4 & Male & 350 & 0.00 \\
& Female & 405 & 0.00 \\
Field-F5 & Male & 251 & 0.00 \\
& Female & 283 & 0.00 \\
Field-F7 & Male & 262 & 0.00 \\
& Female & 292 & 0.00 \\
Field-F9 & Male & 151 & 0.00 \\
& Female & 162 & 0.00 \\
Field-F11 & Male & 135 & 0.00 \\
& Female & 148 & 0.00 \\
Laboratory** & Male & 100 & 100 \\
& Female & 100 & 100 \\
Control*** & & 40 & 0
\end{tabular}

* Mortality refers to the percentage of mosquitoes that died $24 \mathrm{~h}$ after recovery from a $60 \mathrm{~min}$ exposure to the insecticides. Resistance was defined as mortality $<90 \%$, probable resistance was defined as mortality $90-98 \%$, and susceptibility was defined as mortality $>98 \%$. ${ }^{* *}$ The laboratory mosquitoes included the susceptible colony, which has been cultured in the insectaria for more than 500 generations and has never been exposed to any insecticide. ***Forty mosquitoes of laboratory strain were exposed to filter paper without insecticide for $60 \mathrm{~min}$, and mortality was recorded after the $24 \mathrm{~h}$ recovery period. the F3 generation $\left(\mathrm{X}^{2}=0.55, v=1, p>0.05\right)$, and both sexes were completely resistant to deltamethrin after F4. The laboratory $A n$. sinensis mosquitoes, by contrast, were all killed after exposure to the same dose of deltamethrin (Table 1).

To date, this colonised strain has reached its eleventh generation. An sinensis mosquitoes from F1 to F5 were tested with the standard WHO bioassay, followed by the same treatment in F7, F9 and F11, when exhibited stable resistant to the deltamethrin (Table 1).

\section{$k d r$ genotype}

Two types of $k d r$ mutations at codon position 1014 of the para-type sodium channel gene among the randomly selected female field An. sinensis were detected (Table 2): one mutation from TTG to TTT that causes a leucine to phenylalanine substitution (L1014F) and one mutation from TTG to TGT that causes a leucine to cysteine substitution (L1014C). The original population collected from the field (F0) had a high mutation frequency (98.73\%), with a dominant $k d r$ mutation of L1014F. Similarly, all of the subsequent generations from the field F1 to F4, F5 and F7 after the deltamethrin selection exhibited a high mutation frequency, and the individuals in F2, F3, F4, F5 and F7 exhibited a 100\% mutation frequency. The dominant $k d r$ mutation was L1014F in the F3 generation. However, no $k d r$ mutation was detected in the susceptible laboratory strain (Table 2).

\section{Metabolic enzyme activities}

100 and 60 randomly selected female An.sinensis from laboratory susceptible strain and F11, respectively, were tested for metabolic enzyme activities. The median GST activity of the lab strain was $0.231 \mu \mathrm{mol} \mathrm{cDNB} / \mathrm{min} / \mathrm{mg}$ protein (ranging from 0.17 to 0.31 ), and the median $\mathrm{P} 450$ activity was $25.5 \mathrm{pmol} 7-\mathrm{HC} / \mathrm{min} / \mathrm{mg}$ protein (ranging from 19.9 to 31.9 ). Comparing with the lab susceptible strain, significantly elevated levels of both the GSTs and P450s were found in field-selected mosquitoes, F11 (Figure 3). 
Table 2 Distribution of $k d r$ allele frequencies in An. sinensis populations

\begin{tabular}{|c|c|c|c|c|c|c|c|}
\hline \multirow[t]{2}{*}{ Population } & \multirow{2}{*}{$\begin{array}{l}\text { Wild type } \\
\text { TTG/TTG }\end{array}$} & \multicolumn{5}{|l|}{ Mutation } & \multirow{2}{*}{$\begin{array}{l}\text { Mutation } \\
\text { frequency } \\
\text { (\%) }\end{array}$} \\
\hline & & TTT/TTT & TGT/TGT & TTT/TGT & TTG/TGT & $\mathrm{TTG} / \mathrm{TTT}$ & \\
\hline Field-F0 & 0 & 102 & 14 & 38 & 0 & 4 & 98.73 \\
\hline Field-F1 & 0 & 88 & 16 & 48 & 4 & 6 & 96.91 \\
\hline Field-F2 & 0 & 58 & 60 & 24 & 0 & 0 & 100 \\
\hline Field-F3 & 0 & 102 & 0 & 0 & 0 & 0 & 100 \\
\hline Field-F4 & 0 & 70 & 0 & 0 & 0 & 0 & 100 \\
\hline Field-F5 & 0 & 81 & 0 & 0 & 0 & 0 & 100 \\
\hline Field-F7 & 0 & 68 & 0 & 0 & 0 & 0 & 100 \\
\hline Field-F9 & 0 & 72 & 0 & 0 & 0 & 0 & 100 \\
\hline Field-F11 & 0 & 78 & 0 & 0 & 0 & 0 & 100 \\
\hline Laboratory & 63 & 0 & 0 & 0 & 0 & 0 & 0 \\
\hline
\end{tabular}

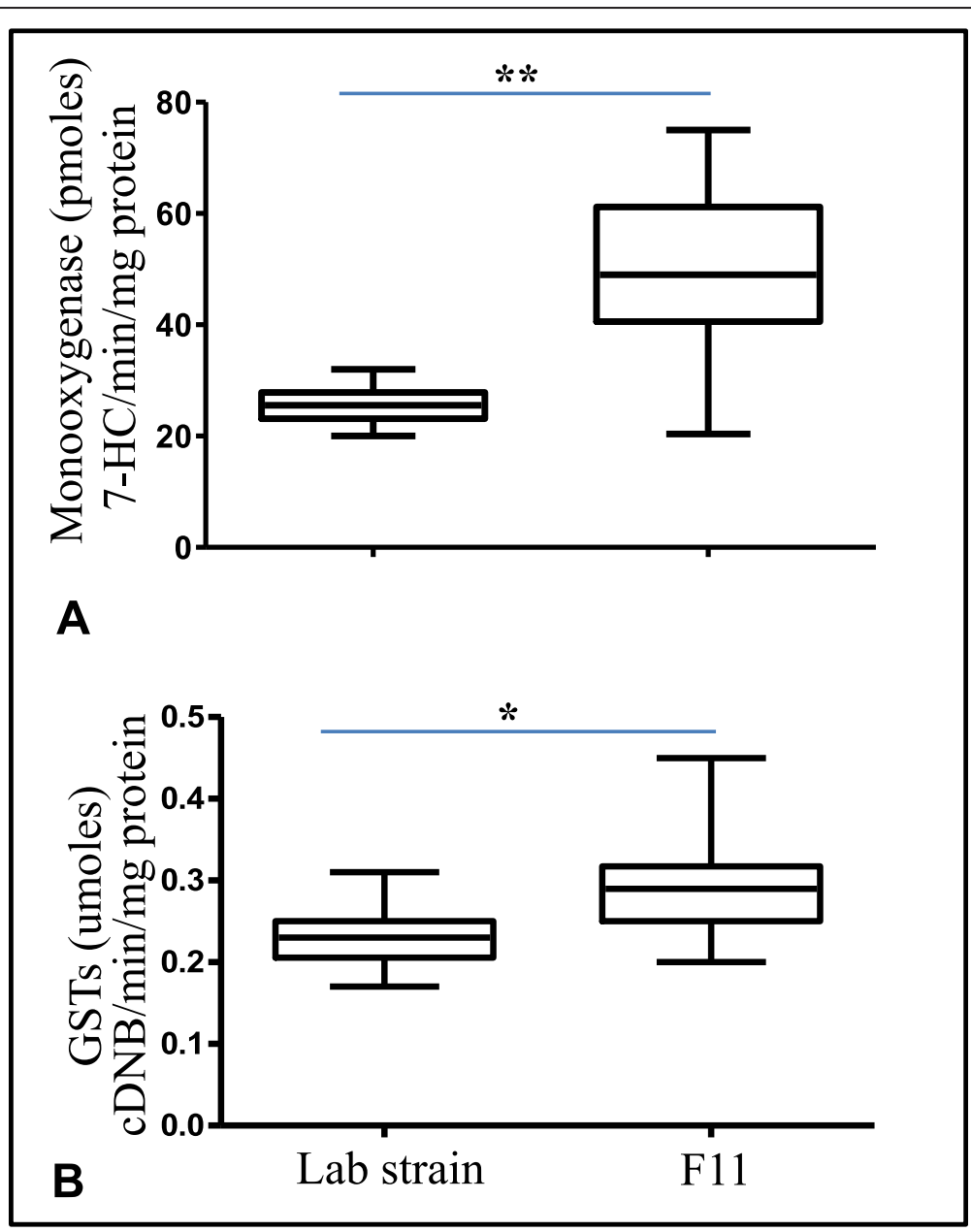

Figure 3 Boxplots of metabolic enzyme activities in An. sinensis from laboratory susceptible strain and field-selected, F11. The median activity is shown by horizontal bar; the upper and lower quartiles is shown by the box, and the full range of the data set is shown by the vertical lines. *,$p<0.05$; ${ }^{*}, p<0.01$. A: P450 monooxygenases; B: GSTs. 


\section{Discussion}

The establishment of resistant strains is essential for further understanding the mechanism of the development of insecticide resistance among insects, including anopheles mosquitoes; the colonised resistant strains could also be used in testing new malaria interventions/tools that might counter physiological resistance among these vector species. As we already possessed the deltamethrin-susceptible strain of An. sinensis in the laboratory, we previously attempted to induce and then select the deltamethrinresistant strain from this colony. However, the median lethal concentrations $\left(\mathrm{LC}_{50} \mathrm{~s}\right)$ after generations of exposure to deltamethrin in 3rd and 4th instar larvae mosquitoes decreased to the original level after only a gradual 1-3-fold increase over several generations. This phenomenon differed greatly from the insecticide selection in other laboratory colonised species, Culex pipiens pallens, in which the LC50 to deltamethrin rapidly increased to approximately fivehundred times the original value after ten continuous generations of exposure [23]. The distinctly different outcomes of deltamethrin selection between An. sinensis and Culex pipiens pallens may be due to the species variation, a possibility that requires further investigation. Compared with the laboratory colony, wild mosquito populations are under continuous selection pressure from insecticide-associated activities related to public health and agriculture $[24,25]$. As a result, the susceptible individuals in these populations decrease while resistant individuals increase [26]. The annual report from vector monitoring and surveillance systems demonstrated that a high level of insecticide resistance in wild populations has developed. Therefore in the present study, we collected wild An. sinensis and applied deltamethrin as a selection agent instead of applying the selection process to the laboratory colony.

To our knowledge, this is the first description of selecting for deltamethrin resistance and maintaining an $A n$. sinensis colony by forced mating. As has been well established, the greatest obstacle to natural mosquito colonisation is the low mating rate due to the switch from the natural environment to the laboratory [27]; the mosquitoes prefer to swarm in open areas rather than mate in narrow cages, especially in the first several generations. Indeed, we failed to colonise this species in 2012 because our specimens failed to produce sufficient progeny after several generations of selection by insecticides. To overcome this difficulty, the forced mating method was used in the present study. The forced mating method is still being used to maintain the colony due to the amount of the population. It is important to note that healthy males and females are critical for successful mating. Other practices can also be helpful, including allowing the engorged female mosquitoes at least $3-5 \mathrm{~h}$ of digestion time prior to mating instead of mating immediately after blood feeding and using males of a similar age or younger than the females. In the addition, although a bloodmeal feeding to females prior to artificial mating may not be necessary, previous research has indicated that the engorged abdomen of the female facilitates forced copulation [28].

Given that the wild mosquitoes already exhibited a high level of resistance to pyrethroids, indicating a very low proportion of susceptible individuals living in the natural populations, we were not surprised to find that the mortality of the progeny of the wild An. sinensis reached zero after only three continuous generations of selection by removing the susceptible individuals in the population. The proportions of both male and female progeny originating from the natural populations tended to increase with deltamethrin resistance after selection by the insecticide. The female cohorts in the first two (F1 and F2) selected generations exhibited markedly higher levels of resistance than their male counterparts, which demonstrated a potential distortion based on the sex-linked factor associated with resistance. However, it should be noted that selection for resistance after the third generation (F3) revealed high levels of survival among females as well as males, which was also supported the results of a previous study on insecticide selection in field-collected An. funestus, which is an important malaria vector in Africa [29].

The mutation at position 1014 causing a change from leucine to either phenylalanine (L1014F) or serine (L1014S), conferring knockdown-resistance $(k d r)$, was the most common among the identified point mutations in the para-type sodium channel gene in anopheline mosquitoes associated with pyrethroid resistance [22,30]. In the present study, the natural population (F0) of An. sinensis included a large subpopulation with the $k d r$ mutation, among which L1014F and L1014C were identified. However, we did not detect any L1014S mutation, unlike previous reports on An. sinensis populations from other regions [31]. Furthermore, the $k d r$ allele (TTG to TTC) leading to L1014F, which was previously observed in central China, was not detected [32], which indicates that the $k d r$ mutation might be largely determined by sampling factors and might differ markedly in different areas. The dominant $k d r$ mutation was the L1014F mutation during the process of selection, with an exception observed only in the F2 generation, consistent with previous results in Culex pipiens pallens mosquitoes subjected to deltamethrin selection [33]. We did not detect any $k d r$ mutation in the laboratory mosquitoes susceptible to deltamethrin compared with the resistant-phenotype field mosquitoes with high $k d r$ mutation status, suggesting that the $k d r$ mutation might play an important role in the formulation of insecticide resistance in this species. However, the continuous increasing level of resistance from F2 to the subsequent generations despite the $100 \% k d r$ mutation frequencies achieved as early as F2 suggests that other issues are also involved in insecticide resistance in addition 
to the $k d r$. In the present study, we found both the levels of enzyme activity in P450s and GSTs were elevated in field insecticide-selected populations, with comparison to laboratory susceptible stain, in which more significantly elevation was found in P450s, supported by the recent studies in the field An. sinensis in central China [34,35], suggesting the metabolic detoxification especially the modification of P450s activity also play important role in the deltamethrin resistance in An. sinensis, besides the $\mathrm{kdr}$ mutation [36,37]. The colonised population of An. sinensis provides a valuable tool for further genetic approaches, e.g. to screen for the major gene loci among P450s conferring the deltamethrin resistance via QTL mapping using this colonised population and the susceptible strain, and to develop the subsequent monitoring method for insecticide resistance detection in this important species.

\section{Conclusions}

The established colonised population of An. sinensis from the field exhibited a high resistance level to deltamethrin with the dominant $k d r$ mutation allele L1014F and elevated detoxification enzyme activities. This population serves as a valuable research tool for future genetic studies in this important malaria vector to further elucidate the mechanism of insecticide resistance, and also for testing new malaria interventions/tools that might counter physiological resistance in this species.

\section{Competing interests}

The authors declare that they have no competing interests.

\section{Authors' contributions}

GZ, JC and QG contributed to the study design. HZ, JL, JT, LB, WW, YG, YL, $F L, Y C, C Z$ and $S X$ contributed to the field mosquito collection and laboratory maintenance. GZ, JL and JT performed the insecticide-resistance bioassays and molecular work. GZ drafted the manuscript. JC and QG revised the manuscript. All authors read and approved the final manuscript.

\section{Acknowledgements}

The authors acknowledge the Yixing County Center for Diseases Control and Prevention (CDC) for the assistance with field mosquito collection. This work was supported by Jiangsu Province's Construction Project (BM2009902), Jiangsu Province's Medical High Tech Platform (ZX201108), National S \& T Major Programme (2012ZX10004220) and Natural Science Foundation of Jiangsu Province (BK20141106 and BK2012106). We are grateful for the support from the Asia Pacific Malaria Elimination Network (APMEN), GZ, YL and $\mathrm{JC}$ were recipients of APMEN fellowships. The funders had no role in the study design, data collection, analysis, decision to publish or preparation of the manuscript.

\section{Author details}

${ }^{1}$ Key Laboratory of Parasitic Disease Control and Prevention (Ministry of Health), and Jiangsu Provincial Key Laboratory of Parasite Molecular Biology, Jiangsu Institute of Parasitic Diseases, Wuxi, Jiangsu Province, People's Republic of China. ${ }^{2}$ Department of Parasitology, Medical College of Soochow University, Suzhou 215123, People's Republic of China. ${ }^{3}$ Public Health Research Center, Jiangnan University, Wuxi, People's Republic of China.

Received: 25 July 2014 Accepted: 30 November 2014

Published online: 11 December 2014

\section{References}

1. Rueda LM, Brown TL, Kim HC, Chong ST, Klein TA, Foley DH, Anyamba A, Smith M, Pak EP, Wilkerson RC: Species composition, larval habitats, seasonal occurrence and distribution of potential malaria vectors and associated species of Anopheles (Diptera: Culicidae) from the Republic of Korea. Malar J 2010, 9:55.

2. Zhou SS, Zhang SS, Wang JJ, Zheng X, Huang F, Li WD, Xu X, Zhang HW: Spatial correlation between malaria cases and water-bodies in Anopheles sinensis dominated areas of Huang-Huai plain. China Parasit Vectors 2012, 5:106.

3. Sinka ME, Bangs MJ, Manguin S, Chareonviriyaphap T, Patil AP, Temperley WH, Gething PW, Elyazar IR, Kabaria CW, Harbach RE, Hay SI: The dominant Anopheles vectors of human malaria in the Asia-Pacific region: occurrence data, distribution maps and bionomic precis. Parasit Vectors 2011, 4:89.

4. Jung J, Jung Y, Min GS, Kim W: Analysis of the population genetic structure of the malaria vector Anopheles sinensis in South Korea based on mitochondrial sequences. Am J Trop Med Hyg 2007, 77(2):310-315.

5. Gao Q, Beebe NW, Cooper RD: Molecular identification of the malaria vectors Anopheles anthropophagus and Anopheles sinensis (Diptera: Culicidae) in central China using polymerase chain reaction and appraisal of their position within the Hyrcanus group. J Med Entomol 2004, 41(1):5-11.

6. Zhu G, Xia H, Zhou H, Li J, Lu F, Liu Y, Cao J, Gao Q, Sattabongkot J: Susceptibility of Anopheles sinensis to Plasmodium vivax in malarial outbreak areas of central China. Parasit Vectors 2013, 6(1):176.

7. Pan JY, Zhou SS, Zheng X, Huang F, Wang DQ, Shen YZ, Su YP, Zhou GC, Liu F, Jiang JJ: Vector capacity of Anopheles sinensis in malaria outbreak areas of central China. Parasit Vectors 2012, 5:136.

8. The malERA Consultative Group on Vector Contro: A research agenda for malaria eradication: vector control. PLOS Med 2011, 8(1):e1000401.

9. Ranson H, N'Guessan R, Lines J, Moiroux N, Nkuni Z, Corbel V: Pyrethroid resistance in African anopheline mosquitoes: what are the implications for malaria control? Trends Parasitol 2011, 27(2):91-98.

10. Chang XL, Xue YQ, Zhang AD, Zhu GD, Fang Q: Deltamethrin resistance, metabolic detoxification enzyme and $k d r$ mutation in Anopheles sinensis in region along Huaihe River in Anhui Province. Zhongguo Xue Xi Chong Bing Fang Zhi Za Zhi 2013, 25(3):263-267.

11. Wang DQ, Xia ZG, Zhou SS, Zhou XN, Wang RB, Zhang QF: A potential threat to malaria elimination: extensive deltamethrin and DDT resistance to Anopheles sinensis from the malaria-endemic areas in China. Malar J 2013, 12:164.

12. Wondji CS, Morgan J, Coetzee M, Hunt RH, Steen K, Black WC, Hemingway J, Ranson H: Mapping a quantitative trait locus (QTL) conferring pyrethroid resistance in the African malaria vector Anopheles funestus. BMC Genomics 2007, 8:34.

13. Ranson H, Paton MG, Jensen B, McCarroll L, Vaughan A, Hogan JR, Hemingway J, Collins FH: Genetic mapping of genes conferring permethrin resistance in the malaria vector, Anopheles gambiae. Insect Mol Biol 2004, 13(4):379-386.

14. Irving $\mathrm{H}$, Riveron JM, Ibrahim SS, Lobo NF, Wondji CS: Positional cloning of rp2 QTL associates the P450 genes CYP6Z1, CYP6Z3 and CYP6M7 with pyrethroid resistance in the malaria vector Anopheles funestus. Heredity (Edinb) 2012, 109(6):383-392.

15. Li Jl, Zhou HY, Cao J, Zhu GD, Wang WM, Gu Y, Liu Y, Cao Y, Zhang C, Gao Q: Sensitivity of Anopheles sinensis to insecticides in Jiangsu Province. Zhongguo Xue Xi Chong Bing Fang Zhi Za Zhi 2011, 23(3):296-300.

16. Ma YJ, Xu JN: The Hyrcanus group of Anopheles (Anopheles) in China (Diptera: Culicidae): species discrimination and phylogenetic relationships inferred by ribosomal DNA internal transcribed spacer 2 sequences. J Med Entomol 2005, 42(4):610-619.

17. WHO: Test procedures for insecticide resistance monitoring in malaria vector mosquitoes. Geneva, Switzerland: World Health Organization; 2013.

18. Amir A, Sum JS, Lau YL, Vythilingam I, Fong MY: Colonization of Anopheles cracens: a malaria vector of emerging importance. Parasit Vectors 2013, 6:81.

19. MR4: Methods in Anopheles Research Second Edition. 2011. (http://www. mr4.org/AnophelesProgram/TrainingMethods.aspx).

20. Bai L, Zhu GD, Tang JX, Zhang C, Liu YB, Li JL, Cao J, Gao Q: Study on TaqMan-MGB real-time fluorescence quantitative PCR to detect gene mutation of $k d r$ from Anopheles sinensis. Zhongguo Xue Xi Chong Bing Fang Zhi Za Zhi 2013, 25(2):167-171. 176.

21. Bai L, Zhu GD, Zhou HY, Tang JX, Li JL, Xu S, Zhang MH, Yao LN, Huang GQ, Wang YB, Zhang HW, Wang SB, Cao J, Gao Q: Development and 
application of an AllGlo probe-based qPCR assay for detecting knockdown resistance (kdr) mutations in Anopheles sinensis. Malar J 2014 13:379.

22. Zhong D, Chang X, Zhou G, He Z, Fu F, Yan Z, Zhu G, Xu T, Bonizzoni M, Wang MH, Cui L, Zheng B, Chen B, Yan G: Relationship between knockdown resistance, metabolic detoxification and organismal resistance to pyrethroids in Anopheles sinensis. PLoS One 2013, 8(2):e55475.

23. Zhu CL, Wang RZ, Gao XH, Ye BH: Seletion of deltamethrin resistant Culex pipiens pallens and Anopheles sinensis. Nan Jing Yi Ke Da Xue Xue Bao 1994, 14(4):3.

24. Nkya TE, Akhouayri I, Kisinza W, David JP: Impact of environment on mosquito response to pyrethroid insecticides: facts, evidences and prospects. Insect Biochem Mol Biol 2013, 43(4):407-416.

25. Abuelmaali SA, Elaagip AH, Basheer MA, Frah EA, Ahmed FT, Elhaj HF, Seidahmed OM, Weetman D, Mahdi Abdel Hamid M: Impacts of agricultural practices on insecticide resistance in the malaria vector Anopheles arabiensis in Khartoum State, Sudan. PLoS One 2013, 8(11):e80549.

26. Wang J: Resistance and response to selection to deltamethrin in Anopheles sinensis from Zhejiang. China J Am Mosa Control Assoc 2000, 16(1):9-12.

27. Klein TA, Lima JB, Toda-Tang A: Colonization and maintenance of Anopheles deaneorum in Brazil. J Am Mosa Control Assoc 1990, 6(3):510-513.

28. Baker RH: Mating problems as related to the establishment and maintenance of laboratory colonies of mosquitoes. Bull World Health Org 1964, 31:467-468.

29. Hunt RH, Brooke BD, Pillay C, Koekemoer LL, Coetzee M: Laboratory selection for and characteristics of pyrethroid resistance in the malaria vector Anopheles funestus. Med Vet Entomol 2005, 19(3):271-275.

30. Ranson $\mathrm{H}$, Jensen B, Vulule JM, Wang X, Hemingway J, Collins FH: Identification of a point mutation in the voltage-gated sodium channel gene of Kenyan Anopheles gambiae associated with resistance to DDT and pyrethroids. Insect Mol Biol 2000, 9(5):491-497.

31. Verhaeghen K, Van Bortel W, Trung HD, Sochantha T, Keokenchanh K, Coosemans M: Knockdown resistance in Anopheles vagus, An. sinensis, An. paraliae and An. peditaeniatus populations of the Mekong region. Parasit Vectors 2010, 3(1):59.

32. Tan WL, Li CX, Wang ZM, Liu MD, Dong YD, Feng XY, Wu ZM, Guo XX Xing D, Zhang YM, Wang ZC, Zhao TY: First detection of multiple knockdown resistance (kdr)-like mutations in voltage-gated sodium channel using three new genotyping methods in Anopheles sinensis from Guangxi Province, China. J Med Entomol 2012, 49(5):1012-1020.

33. Chen L, Zhong D, Zhang D, Shi L, Zhou G, Gong M, Zhou H, Sun Y, Ma L, He J, Hong S, Zhou D, Xiong C, Chen C, Zou P, Zhu C, Yan G: Molecular ecology of pyrethroid knockdown resistance in Culex pipiens pallens mosquitoes. PLoS One 2010, 5(7):e11681.

34. Chang X, Zhong D, Fang Q, Hartsel J, Zhou G, Shi L, Fang F, Zhu C, Yan G. Multiple Resistances and Complex Mechanisms of Anopheles sinensis Mosquito: A Major Obstacle to Mosquito-Borne Diseases Control and Elimination in China. PLoS Negl Trop Dis 2014, 8(5):e2889.

35. Zhu G, Zhong D, Cao J, Zhou H, Li J, Liu Y, Bai L, Xu S, Wang MH, Zhou G, Chang X, Gao Q, Yan G: Transcriptome profiling of pyrethroid resistant and susceptible mosquitoes in the malaria vector, Anopheles sinensis. BMC Genomics 2014, 15(1):448.

36. Chen H, Githeko AK, Githure Jl, Mutunga J, Zhou G, Yan G: Monooxygenase levels and knockdown resistance $(k d r)$ allele frequencies in Anopheles gambiae and Anopheles arabiensis in Kenya. J Med Entomol 2008, 45(2):242-250.

37. Hemingway J, Hawkes NJ, McCarroll L, Ranson H: The molecular basis of insecticide resistance in mosquitoes. Insect Biochem Mol Biol 2004, 34(7):653-665

doi:10.1186/s13071-014-0582-7

Cite this article as: Zhu et al: The colonization of pyrethroid resistant strain from wild Anopheles sinensis, the major Asian malaria vector. Parasites \& Vectors 2014 7:582.

\section{Submit your next manuscript to BioMed Central and take full advantage of:}

- Convenient online submission

- Thorough peer review

- No space constraints or color figure charges

- Immediate publication on acceptance

- Inclusion in PubMed, CAS, Scopus and Google Scholar

- Research which is freely available for redistribution
C Biomed Central 